\title{
Editorial
}

\section{Un reto para Colombia: mejorar la utilización de medicamentos probadamente beneficiosos en la prevención secundaria cardiovascular}

Las enfermedades cardiovasculares (ECV) causan 30\% del total de las muertes en todo el mundo; de estas $80 \%$ sucede en los países en vía de desarrollo ${ }^{1}$. El 1\% de las personas en el mundo presenta un evento coronario agudo o cerebrovascular por año y la mitad de estos eventos se produce en individuos con enfermedad vascular preexistente ${ }^{2}$. Los países de bajos y medianos ingresos presentan un incremento en la carga de las ECV y la mayoría no tiene programas concretos y bien definidos para su prevención ${ }^{3}$.

Se ha estimado que para el año 2020, habrá un aumento entre $120 \%$ y $137 \%$ en la incidencia de enfermedad coronaria en las mujeres y los hombres respectivamente, en los países en desarrollo ${ }^{4}$. Este fenómeno se explica, entre otras razones, por la denominada transición epidemiológica, que se asocia con un notable aumento en la prevalencia de factores de riesgo como la obesidad, la hipertensión arterial, el tabaquismo, el consumo exagerado de alcohol, los cambios en los hábitos nutricionales y el sedentarismo. Estos factores están relacionados con la rápida urbanización que han sufrido los países de bajos y medianos ingresos ${ }^{5,6}$.

El gasto en salud per cápita en los países de bajos y medianos ingresos no alcanza el 10\% de la cantidad que se invierte en atención en salud en los países de altos ingresos ${ }^{7}$. Esta situación, así como el estricto control presupuestario de los sistemas de salud de los países de bajos y medianos ingresos obliga a tener políticas de salud pública eficientes, que deben priorizar las intervenciones preventivas y curativas basadas en estudios que generen una indiscutible evidencia. Por esto se propone que la prevención de las ECV en Colombia debe ofrecer al menos un adecuado programa de prevención secundaria, es decir, garantizar que el Sistema General de Seguridad Social en Salud ofrezca un manejo eficiente de los individuos con cardiopatía coronaria, enfermedad cerebrovascular o vasculopatía periférica establecida, lo que redundaría en la reducción de eventos agudos y de muerte prematura tanto en las personas con ECV establecida como en aquellas con alto riesgo cardiovascular $^{8,9}$. La evidencia es contundente porque el tratamiento farmacológico (antiplaquetarios, beta bloqueadores, estatinas e inhibidores del sistema renina-angiotensina tipo IECAS o ARAS) en estos pacientes reduce el riesgo de nuevos eventos cardiovasculares $^{10-13}$.

En los países de altos ingresos estas intervenciones farmacológicas demostraron una disminución de la mortalidad por ECV; sin embargo, el escenario es desolador en los países de bajos y medianos ingre$\operatorname{sos}^{14}$ porque no existen datos del impacto de programas de prevención secundaria de ECV con la utilización de las 4 familias de medicamentos que se han mostrado beneficiosos en la reducción de la morbimortalidad por estas enfermedades, a pesar de la existencia de una serie de reportes en los que se propone el costo-beneficio que tendrían estos programas preventivos. Así, por ejemplo, Lim et al. ${ }^{15}$ informaron que el aumento de la cobertura del uso conjunto de medicamentos en prevención secundaria cardiovascular (aspirina, estatina y dos medicamentos hipotensores) podría evitar 17.9 millones de muertes por ECV (IC 95\%: 7.4-25.7 millones). Además, se obtendría una disminución del $56 \%$ de las muertas evitables en los menores de 70 años y en las mujeres. El costo anual promedio per cápita de esta intervención sería de U\$1.08 (IC 95\%: 0.75-1.40) en Estados Unidos, pero solo de U\$0.43 a U\$0.90 en países de bajos ingresos y de U\$0.54 a U\$2.93 en los de medianos ingresos ${ }^{15}$, lo que facilitaría el uso de medicamentos en la prevención secundaria de las ECV, sobre todo la enfermedad cardíaca isquémica y la enfermedad cerebrovascular.

Con el objeto de profundizar en el conocimiento de la situación de la prevención secundaria cardio- 
vascular en el contexto mundial y en los países no desarrollados en general y en particular Colombia, recientemente se publicó los primeros resultados ${ }^{16}$ del estudio Prospective Urban RuralEpidemiology (PURE) liderado por elDr. Salim Yusuf del Population Health Research (PHRI) de la Universidad de MacMaster en Canadá. En el estudio se incluyeron 628 comunidades con un total del 153,996 adultos de 35 a 70 años de áreas urbanas y rurales de países de altos ingresos (Canadá, Suecia y Emiratos Árabes Unidos), de medianos altos (Argentina, Brasil, Chile, Malasia, Polonia, Sudáfrica y Turquía), de medianos bajos (China, Colombia e Irán) y de bajos ingresos (Bangladesh, India, Paquistán y Zimbabwe). En Colombia se incluyeron 7,500 individuos de 13 departamentos. Se demostró un bajo uso de medicamentos en prevención secundaria en los países de bajos y medianos ingresos en comparación con los países de altos ingresos, lo que ratifica la gran brecha existente en prevención secundaria cardiovascular en el mundo.

Así se observa que apenas $25.1 \%$ de los individuos con antecedentes de infarto agudo de miocardio (5,650 participantes) o accidente cerebrovascular (2,292 participantes) provenientes de los países de medianos y bajos ingresos estaba utilizando antiplaquetarios (aspirina), 18.4\% beta bloqueadores, $18.3 \%$ inhibidores de la enzima convertidora de angiotensina o antagonistas de los receptores de la angiotensina, 10.7\% un diurético, y 13.3\% estatinas. En los países de ingresos medios altos, 45.1\% no recibía medicamento y estos porcentajes se incrementaron a $69.3 \%$ en los países de medios bajos ingresos y a $80.2 \%$ en los países de bajos ingresos. En el Cuadro 1 se presentan los resultados especificados por regiones geográficas (Suramérica) y por ingresos (altos y bajos) ajustado por edad. El uso de medicamentos fue mayor en las zonas urbanas que en las rurales y existió una fuerte correlación entre las tasas totales de uso de medicamentos y el gasto por persona en salud en los países estudiados.

Adicionalmente, los pacientes con accidente cerebrovascular tenían una menor probabilidad de recibir medicamentos eficaces (por ejemplo estatinas), en comparación con aquellos con eventos coronarios. En términos globales, en los países de medianos y bajos ingresos $58 \%$ de los pacientes con enfermedad coronaria y $50 \%$ con ECV no recibían ninguno de estos medicamentos de bajo costo y probadamente

\section{Cuadro 1}

Porcentaje de uso de medicamentos de los individuos con enfermedad cardíaca isquémica o enfermedad cerebrovascular ajustado por edad del total por estatus económico por países y región

\begin{tabular}{lcccc}
\hline \multirow{2}{*}{ Medicamentos } & \multicolumn{4}{c}{$\%$} \\
\cline { 2 - 5 } & $\begin{array}{c}\text { Países con } \\
\text { altos ingresos }\end{array}$ & América del Sur & $\begin{array}{c}\text { Países con } \\
\text { bajos ingresos }\end{array}$ & Total \\
\hline Antiplaquetarios & 59.0 & $\mathbf{2 8 . 8}$ & 9.1 & 25.1 \\
Beta bloqueadores & 38.1 & $\mathbf{2 8 . 8}$ & 10.0 & 18.4 \\
IECA/ARA* & 46.6 & $\mathbf{3 7 . 9}$ & 5.3 & 18.3 \\
Diuréticos & 14.1 & $\mathbf{1 9 . 5}$ & 3.7 & 10.7 \\
Bloqueadores de canales de calcio & 17.8 & $\mathbf{1 0 . 8}$ & 6.3 & 11.7 \\
Para bajar presión arterial & 70.9 & $\mathbf{5 8 . 7}$ & 19.8 & 43.2 \\
Estatinas & 64.0 & $\mathbf{1 4 . 6}$ & 3.4 & 13.3 \\
\hline
\end{tabular}

* IECA/ARA: Inhibidores de la enzima convertidora de angiotensina o antagonistas de los receptores de la angiotensina 
eficaces para disminuir la morbi-mortalidad porECV. El bajo uso de medicamentos para prevención secundaria no solo se limitó a los países con bajos y medianos ingresos, sino que también se observó que en países de altos ingresos como Canadá, Suecia y Emiratos Árabes Unidos, no se alcanzaron coberturas del $100 \%$, y se encontró que un número significativo de pacientes $(11.6 \%)$ con enfermedad coronaria y enfermedad cerebrovascular no tenía ningún tratamiento preventivo ${ }^{16}$.

Es necesario resaltar que los medicamentos eficaces para prevención secundaria son de muy bajo costo y, sin embargo, como el estudio PURE lo demuestra, su uso es muy limitado en países como Colombia. Así, a pesar de que es de amplio conocimiento que el uso de aspirina es efectiva en la reducción del riesgo de nuevos eventos coronarios y de accidentes cerebrovasculares en hombres y mujeres con antecedentes de ECV y que es extremadamente económica, su uso alcanzó a cubrir 28\% de los pacientes suramericanos con antecedentes de ECV. Por lo tanto, es prioritario identificar cuáles son las causas en Colombia que determinan tan bajo porcentaje de usuarios en prevención secundaria a pesar de que su uso está recomendado en las guías terapéuticas vigentes ${ }^{17}$.

Las limitaciones para la implementación de las estrategias farmacológicas de prevención secundaria en Colombia son similares a las observadas en otros países de bajos y medianos ingresos y que sugieren que pueden estar relacionadas con políticas públicas sanitarias inadecuadas, con el bajo acceso a la atención en salud cardiovascular y a la falta de medicamentos asequibles en relación con las coberturas de los sistemas de salud, así como con la baja adherencia a los tratamientos propuestos, entre otras causas por la necesidad de tomar al menos 4 diferentes medicamentos ${ }^{18}$. Tampoco existen en Colombia estudios que evalúen la eficacia y seguridad de las denominadas poli-píldoras o poli-cápsulas que integran en una sola presentación los 4 fármacos esenciales para la prevención secundaria de las ECV ${ }^{14}$. También habrá que estudiar si la conducta de los médicos y otros miembros del equipo de salud es lo suficientemente rigurosa en términos de prescribir y recomendar una adecuada adherencia a los tratamientos medicamentosos preventivos recomendados.

Son escasos los estudios de costo-efectividad de las estrategias de prevención secundaria en los países en desarrollo, y es necesario tener precaución con la transferencia de las estimaciones de la efectividad de los programas de prevención secundaria en ECV de los países desarrollados, porque los aspectos de rentabilidad en los países de bajos y medianos ingresos están influidos por aspectos no solo económicos, sino también socioculturales ${ }^{19}$.

En conclusión, para los países de bajos y medianos ingresos en general, y para Colombia en particular, un reto inmediato que debe ser asumido por los actores involucrados en el cuidado de la salud es fortalecer la capacitación de los profesionales de salud sobre el uso de medicamentos eficaces y económicos para la prevención secundaria de las enfermedades cardiovasculares. Además, se debe realizar estudios que permitan determinar con veracidad las causas para el bajo uso de medicamentos eficaces y económicos existentes en el arsenal terapéutico colombiano, y tomar las acciones correctivas.

Es oportuno demostrar que el costo anual promedio per cápita del uso de medicamentos efectivos que impactan en la historia natural de las enfermedades cardiovasculares es más costo-efectivo que continuar con el manejo de un modelo expectante que cubre los altos costos que demandan los eventos agudos de la enfermedad cardiovascular. Más aún, la prevención secundaria de las ECV se debe complementar con acciones de prevención primaria con cambios en los estilos de vida y el ejercicio físico controlado. Finalmente, una política pública sana para los países de bajos y medianos ingresos, debería contemplar intervenciones de prevención primordial que permita actuar en forma eficiente sobre las causas de los factores de riesgo emergentes ligados con la urbanización. 


\section{Referencias}

1. Mathers CD, López A, Stein C, Ezzati M, Jamison, DT, Murray, CJ. Deaths and disease burden by cause: global burden of disease estimates for 2001. Bethesda: World Bank Country Groups, Disease Control Priorities Project; 2005.

2. Yusuf S, Reddy S, Óunpuu S, Anand S. Global burden of cardiovascular diseases. Part I: General considerations, the epidemiologic transition, risk factors, and impact of urbanization. Circulation. 2001; 104: 2746-53.

3. Fuster VJ, Voûte J. Chronic diseases are not on the agenda. Lancet. 2005; 366: 1512-14.

4. Leeder S, Raymond S, Greenberg H, hasta 6 autores et al. A race against time: The challenge of cardiovascular disease in developing economies. New York: Trustees of Columbia University; 2004.

5. López-Jaramillo P, Silva SY, Rodríguez-Salamanca N, Duran A, Mosquera W, Castillo V. Are nutrition-induced epigenetic changes the link between socioeconomic pathology and cardiovascular diseases? Am J Ther. 2008; 15:362-72.

6. López-Jaramillo P. Defining the research priorities to fight the burden of cardiovascular diseases in Latin America. $J$ Hypertens. 2008; 26: 1886-9.

7. World Bank. World development indicators. Washington, DC: World Bank; 2010.

8. WHO. Prevention of cardiovascular disease. Guidelines for Assessment and management of total cardiovascular risk. Geneva: World Health Organization; 2007.

9. WHO. Prevention of recurrent heart attacks and strokes in low and middle income populations: evidence-based recommendations for policy-markers and health professionals. Geneva: World Health Organization; 2003.

10. Brady AJB, Oliver MA, Pittard JB. Secondary prevention in 24,431 patients with coronary heart disease: survey in primary care. BMJ. 2001; 322: 1463.

11. Wald NJ, Law MR. A strategy to reduce cardiovascular disease by more $\tan 80 \%$. BMJ. 2003; 326: 1419 .
12. Yusuf $S$. Two decades of progress in preventing vascular disease. Lancet. 2002; 360: 2-3.

13. Murray CJL, Lauer JA, Hutubessy RC. Effectiveness and costs of interventions to lower systolic blood pressure and cholesterol: a global and regional analysis on reduction of cardiovascular-disease risk. Lancet. 2003: 361: 717-25.

14. Sanz G, Fuster V. Polypill and global cardiovascular health strategies. Semin Thorac Cardiovascular Surg. 2011; 23: 24-9.

15. Lim SS, Gaziano TA, Gakidou E, Reddy KS, Farzadfar F, Lozano R, et al. Prevention of cardiovascular disease in high-risk individuals in low-income and middle-income countries: health effects and costs. Lancet. 2007; 370:205462.

16. Yusuf S, Islam S, Chow CK, Rangarajan S, Dagenais G, Diaz R, et al. On behalf of the Prospective Urban Rural Epidemiology (PURE) Study Investigators. Use of secondary prevention drugs for cardiovascular disease in the community in high-income, middle-income, and lowincome countries (the PURE study): a prospective epidemiological survey. Lancet. 2011; 378: 1231-43.

17. García RG, López-Jaramillo P. Uso de aspirina en la prevención de enfermedades cardiovasculares. Rev Colom Cardiol. 2008; 15: 223-30.

18. Suhrcke M, Boluarte TA, Niessen L. A systematic review of economic evaluations of interventions to tackle cardiovascular disease in low-and middle-income countries. MBC Public Health. 2012; 12: 2

19. Geoffrey R, Tee K, Marmot M. Rose's strategy of preventive medicine. Oxford: Oxford University Press; 1992.

\section{Patricio López-Jaramillo, $M D^{1,2}$ Paul Anthony Camacho, $\mathbf{M D}^{2}$}

1 Director de Investigaciones, Desarrollo e Innovación Tecnológica, Fundación Oftalmológica de Santander (FOSCAL), Bucaramanga,Colombia.e-mail: investigaciones@foscal.com.co Facultad de Medicina, Universidad de Santander, Bucaramanga, Colombia. 